\title{
Rosazea-artige kutane Infiltration des Gesichtes (Facies leontina) bei chronisch-lymphatischer Leukämie
}

\section{Zusammenfassung}

Wir berichten von einem 77-jährigen Patienten, bei dem rosazea-artige Hautveränderungen im Gesicht auftraten. Die Histologie zeigte ein kutanes B-Zell-Infiltrat der seit 6 Jahren bekannten chronisch-lymphatischen Leukämie. Bei rosazea-artigen Hautveränderungen im Gesicht sollte insbesondere bei älteren Menschen immer auch an eine zugrunde liegende Leukämie gedacht werden.

\section{Abstract}

We report on a 77-year-old male patient with leukaemia cutis presenting as rosacea-like infiltration of the face. Histology and immunostaining showed a dense dermal B-cell infiltrate diagnostic for leukaemia cutis. Whenever especially older patients suffer from rosacea-like nodules and erythema of the face a leukaemia cutis should be considered.

\section{Einleitung}

Die chronisch-lymphatische Leukämie ist mit einer Inzidenz von 3/100 000 die häufigste Leukämieform in Westeuropa. Der Altersgipfel liegt zwischen dem 65. und dem 70. Lebensjahr. Leukämien können mit verschiedenen spezifischen und unspezifischen Hautveränderungen einhergehen, wobei insgesamt spezifische Hautveränderungen seltener sind und bei chronischen Leukämien häufiger auftreten als bei akuten Leukämien.

\section{Kasuistik}

\section{Anamnese}

Bei Erstvorstellung im November 2004 berichtet der 77-jährige Rentner, dass er erstmals im November 2003 eine Rötung und Knötchen im Bereich der Nase bemerkt habe. Unter Anwendung von mehreren nicht mehr eruierbaren Externa sei es zu einer Zunahme und knotigen Veränderung im Bereich der Nase, der Wangen und der Augenbrauen sowie im Verlauf der Ohren ge- kommen (Abb.1). Erst ein Jahr später habe er erstmals einen Hautarzt aufgesucht, welcher ihn direkt in die Klinik weitergeleitet habe.

An Grunderkrankungen sei seit 1998 eine chronisch lymphatische Leukämie bekannt, welche er rein homöopathisch mit Kräutertee und Globuli sowie pflanzlichen Entgiftungskuren behandle.

Aufgrund der Hautveränderungen und unter Berücksichtigung der Anamnese führten wir neben der Labordiagnostik eine Probeexzision zur histologischen und immun-histologischen Diagnosesicherung durch.

\section{Befunde}

\section{Körperliche Untersuchung}

Bei Aufnahme sah man im Gesicht insbesondere über den Augenbrauen, an den Wangen und den Ohren multiple, weiche,

Institutsangaben

Klinik für Dermatologie und Allergologie, Zentrum für Hautkrankheiten, Klinikum Stuttgart

(Direktor: Prof. Dr. P. von den Driesch) 


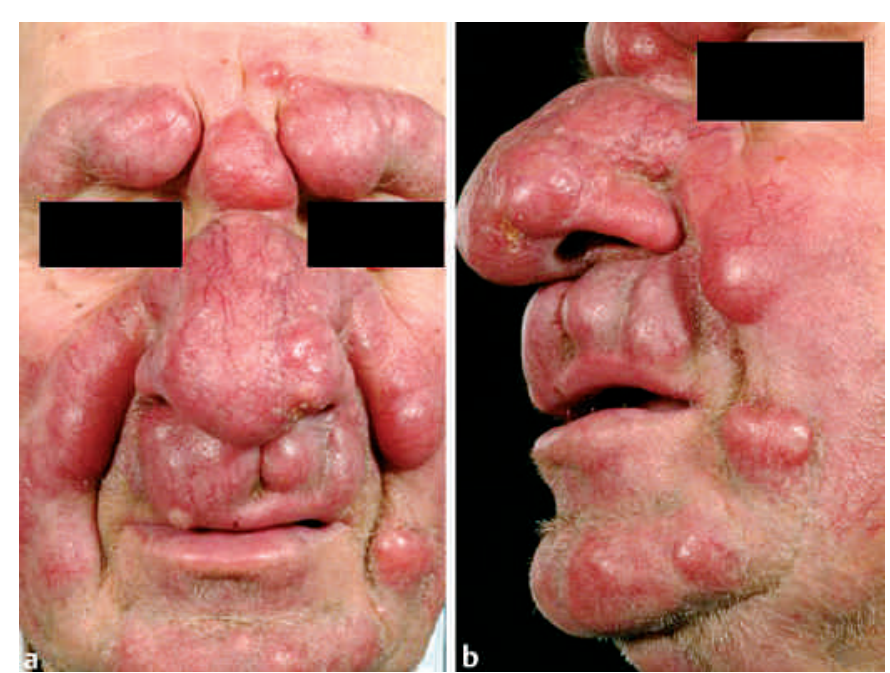

Abb. 1 a,b Knotiges rosazea-artiges kutanes Infiltrat der chronischlymphatischen Leukämie.

erythematöse Knoten. Die Nase war rhinophym-artig erythematös infiltriert mit deutlich erkennbaren Teleangiektasien und bereits eingeschränkter Nasenatmung (Abb.1). Lymphknoten waren zervikal, axillär und inguinal beidseits deutlich vergrößert tastbar. Außerdem war die Milz palpabel.

\section{Histologie}

Die (Immun-) Histologie passte zu einem spezifischen Infiltrat bei bekannter chronisch lymphatischer Leukämie mit Orthokeratose und abgeflachter Epidermis ohne atypische Exozytose. Subepithelial war die gesamte Adnexe umgeben mit einem monomorph wirkenden Infiltrat aus lymphozytären Tumorzellen, welche überwiegend für den B-Zell-Keimzentrumsmarker L26 positiv waren und eine hohe Proliferation sowie einzelne atypische Mitosen zeigten (Abb. 2).

\section{Laboruntersuchungen}

Kleines Blutbild mit Differenzialblutbild: Erhöht waren: Leukozyten $55800 / \mu \mathrm{l} \quad(4000-10000 / \mu \mathrm{l}), \quad$ Lymphozyten $88,3 \%$ (18-48\%), basophile Granulozyten 1,8\% (<1,5\%), stabkernige Granulozyten $14 \%$ (<3\%). Erniedrigt waren: Hämoglobin $12,9 \mathrm{mg} / \mathrm{dl}, \quad$ Hämatokrit $39,5 \% \quad(40-50 \%)$ Erythrozyten $4,3 \times 10^{6} / \mu \mathrm{l} \quad\left(4,5-5,9 \times 10^{6} / \mu \mathrm{l}\right.$, Thrombozyten $116000 / \mu \mathrm{l}$ $(150000-350000 / \mu \mathrm{l})$, neutrophile Granulozyten 7,6\% (40-75\%), Segmentkernige 20\% (50-70\%), Monozyten 1,9\% (4-11\%), eosinophile Granulozyten 0,4\% (<8,4\%). Normwertig waren: MCV, MCH, MCHC. Deutlich erkennbar Gumprecht'sche Zellschatten. Standardserumanalyse: Normwertig waren: Glukose, Harnstoff, Kreatinin, Harnsäure, Natrium, Kalium, Kalzium, Gesamt-Eiweiß, LDH, GOT, GPT, $\gamma$-GT, CRP. Eiweiß-Elektrophorese: normwertig waren: A1-Globulin, A2-Globulin, Beta-Globulin, Gamma-Globulin. Immunfixations-Elektrophorese: erhöht war: Freie Leichtkette Kappa 25,0 mg/dl (3,3-19,4 mg/dl). Normwertig war: freie Leichtkette Lambda. Urinstatus: negativ waren: Bilirubin, Urobilirubin, Ketonkörper, Glukose, Eiweißstix, Blut, Nitrit, Leukozyten. Normwertig waren: spezifisches Gewicht, PhWert. FACS-Analyse: Pan B-Zellen (CD19+) 94,7\%, B-Zellen (HLADR+/CD3-) 95,1\%, B-CLL-Zellen (CD5+/CD20+) 94,8\%, B-Zellen (CD20+/CD21+) 77,1\%, Vorläufer B-/reife B-Zellen (prä-B-ALL) 0,6\%, (Pro-)B-Lymphozyten (FMC7) 0,7\%.

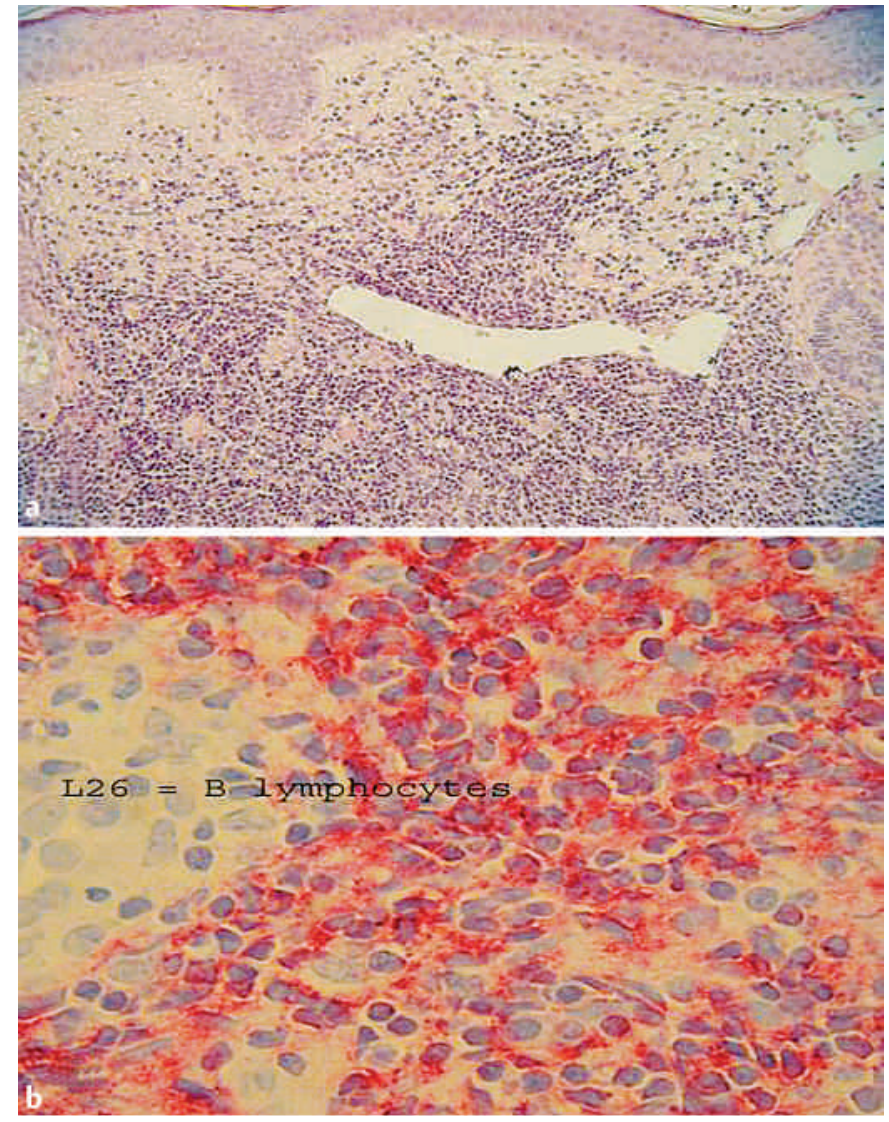

Abb. 2 a Histologie mit monomorpher Durchsetzung der gesamten Cutis mit hyperchromatischen homogenen kleinkernigen Lymphozyten, $\times 50$. b Immunhistologie mit Tumorzellen, durchweg positiv für den B-Zell-Marker L26× 150 .

\section{Sonderuntersuchungen}

In der durchgeführten Lymphknotensonographie sah man Lymphome zervikal, axillär und inguinal beidseits. Die Röntgen-Thorax-Aufnahme zeigte Lymphome hilär beidseits. In der Abdomensonographie sah man eine Hepatosplenomegalie. Das Thorax-Abdomen-CT zeigte parahiläre und abdominale Lymphome.

\section{Therapie und Verlauf}

Nach den Empfehlungen der Deutschen CLL-Studiengruppe [1] leiteten wir eine Therapie mit 0,4 mg/kg Körpergewicht Chlorambucil in 14-tägigen Abständen ein. Zusätzlich verabreichten wir Prednisolon über 3 Tage in absteigender Dosierung $75 \mathrm{mg} /$ $50 \mathrm{mg} / 25 \mathrm{mg}$ nach Knospe.

\section{Diskussion}

Wir berichten über einen Patienten mit rosazea-artigem kutanem Infiltrat einer chronisch-lymphatischen Leukämie.

Hautveränderungen bei Leukämien sind oft beschrieben. Häufiger sind dabei unspezifische Hauterscheinungen wie Sweet-Syndrom, Urtikaria, leukozytoklastische Vaskulitis und Erythema nodosum. Des Weiteren kommen komplizierend zur Grunderkrankung aufgrund des gestörten Immunsystems bakterielle, virale oder Pilzinfektionen der Haut vor [2]. Spezifische lymphozytäre Infiltrate bei Leukämien sind meist Papeln und Knötchen, 
Plaques, Makulae, Ulzerationen oder palpable Purpura [3]. In einzelnen Fällen sind kutane Infiltrate das primäre Symptom, das zur Diagnosestellung führt [4]. Diffuse erythematöse, knotige, rosacea- oder facies leontina-artige Infiltrate im Gesicht sind bisher nur in 2 Fällen beschrieben worden, wobei die Patienten im einen Fall eine zugrundeliegende T-Zell-Leukämie [5] und im anderen Fall eine akute myeloische Leukämie [6] hatten.

Differenzialdiagnostisch kam neben einem primären B-ZellLymphom ein Pseudolymphom, eine Sarkoidose (Lupus pernioTyp) oder ein Lupus vulgaris in Frage.

Histologisch ließ sich das homogene L-26-positive Infiltrat eindeutig von einem primären B-Zell-Lymphom oder einem Pseudolymphom abgrenzen, da es sich um ein monomorphes Infiltrat aus atypischen, kleinen, zytoplasmaarmen Lymphozyten handelte. Eine Sarkoidose oder ein Lupus vulgaris kamen aufgrund der Histologie nicht in Frage.

Fraglich ist, ob in unserem Fall nicht ursprünglich eine Rosazea bestand, die - ähnlich wie in der Literatur für Herpesviren beschrieben [7] - als Triggerfaktor für die Einwanderung der B-Lymphozyten in die Haut fungierte.

Die Überprüfung der Laborparameter bestätigte bei erhöhten Leukozyten und verminderten Erythrozyten und Thrombozyten sowie deutlich erkennbaren Gumprecht'schen Zellschatten in den Leukozyten erneut die vor 6 Jahren gestellte Diagnose einer chronisch lymphatischen Leukämie. Die durchgeführte FACSAnalyse zeigte das typische Bild von CD5-, CD20- und CD23-positiven sowie FMC7- und CD79 $\beta$-negativen B-Lymphozyten.

Beim durchgeführten Staging sah man neben dem Hautbefund eine tastbare und sonographische Lymphknotenbeteiligung zervikal, axillär und inguinal beidseits sowie ein Hepatosplenomegalie und hiläre Lymphknotenbeteiligung beidseits. Aufgrund der $\mathrm{Hb}$ - und Thrombozytenwerte ließ sich die chronische B-ZellLeukämie nach Binet [8] ins Stadium B einklassifizieren. Von einer Knochenmarksbiopsie sahen wir zunächst ab, da diese zur Diagnosesicherung nicht erforderlich ist [1].

Therapeutisch hat sich in den letzten Jahren bei der Behandlung der chronisch lymphatischen Leukämie viel getan, die Therapie der ersten Wahl ist jedoch immer noch Chlorambucil $(0,4 \mathrm{mg} / \mathrm{kg}$ KG alle 14 Tage mit einer Dosissteigerung um 0,1 mg/kg KG pro
Zyklus bis zu einer Enddosis von 0,8 mg/kg KG oder 0,07 mg$0,1 \mathrm{mg} / \mathrm{kg}$ KG täglich für 14 Tage, nächster Zyklus nach 15 Tagen Therapiepause), bei möglichen autoimmunologischen Komplikationen ist die zusätzliche Gabe von Prednisolon in absteigender Dosierung über 3 Tage (75-50-25 mg) sinnvoll. Diese Therapie sollte für 8-12 Monate bis zum maximalen Ansprechen durchgeführt werden. Bei erfolgreicher Behandlung kann nach 12-monatiger Pause bei Progress ein erneuter Zyklus erfolgen. Neuere sehr erfolgreiche Therapieansätze sind die Anwendung von Purinanaloga (Fludarabin, Cladibrin) oder von monoklonalen Antikörpern (CD20 Rituximab, CD52 Alemtuzumab) [1].

Die spezifischen Infiltrate können zusätzlich mit schnellen Elektronen bestrahlt werden oder operativ entfernt werden [9].

\section{Fazit für die Praxis}

Zusammenfassend sollte bei knotig-erythematösen teleangiektatischen Hautveränderungen im Gesicht insbesondere bei älteren Patienten immer eine zugrunde liegende Leukämie mitbedacht werden.

\section{Literatur}

${ }^{1}$ Hallek M, Bergmann M, Emmerich B. Chronic lymphatic leukemia: Up-dated recommendations on diagnosis and treatment. Onkologie 2004; 27: 97-104

2 Dreizen S. The many faces of adult leukemia. Compendium 1991; 12: 46, $48-51$

${ }^{3}$ Su WP, Buechner SA, Li CY. Clinicopthologic correlations in leukaemia cutis. J Am Acad Dermatol 1984; 11: $121-128$

${ }^{4}$ Schneider LA, Weber L, Viardot A, Schubert R, Hinrichs R, ScharffetterKochenek K. Cutaneous leukaemic ifiltrations in a patient with previosly undiagnosed myelodysplastic syndrome. Clin Exp Dermatol. 2004; 29: $468-470$

${ }^{5}$ Miyoshi I, Sumida M. Skin involvement in adult T-cell leucemia. Br J Haematol 2000; 109: 465

${ }^{6}$ Fadilah SA, Alawiyah AA, Amir MA, Cheong SK. Leukemia cutis presenting as leonine facies. Med J Malaysie 2003; 58: $102-104$

${ }^{7}$ Wakelin SH, Young E, Kelly S, Turner M. Transient leukaemia cutis in chronic lymphatic leukaemia. Clin Exp Dermatol 1997; 22: 37-40

${ }^{8}$ Binet JL, Auquier A, Dighiero G, Chastang C, Piguet H, Goasguen J, Vaugier G, Potron G, Colona P, Oberling F, Thomas M, Tchernia G, Jacquillat C, Boivin P, Lesty C, Duault MT, Monconduit M, Belabbes S, Gremy F. A new prognostic classification of chronic lymphocytic leukemia derived from a multivariate survival analysis. Cancer 1981; 48: 98-206

${ }_{9}^{9}$ Baer MR, Barcos M, Farrell H, Raza A, Preisler HD. Acute myelogenous leukemia with leukemia cutis. Eighteen cases seen between 1969 and 1986. Cancer 1989; 63: $2192-2200$ 